\title{
Features of Ontogeny of Wheat Hybrid of Type Dwarf II
}

\section{Ruzanna Robert Sadoyan}

Scientific Center of Agriculture, Ministry of Agriculture, Echmiadzin, Republic of Armenia

\section{Email address:}

ruzannasad@mail.ru

\section{To cite this article:}

Ruzanna Robert Sadoyan. Features of Ontogeny of Wheat Hybrid of Type Dwarf II. Agriculture, Forestry and Fisheries. Vol. 4, No. 3, 2015, pp. 101-105. doi: 10.11648/j.aff.20150403.14

\begin{abstract}
The genes of hybrid depression widely spread in the genus Triticum lead to inviability of hybrid plants and prevent the successful implementation of breeding programs. At the same time the phenomenon of hybrid depression serves as a basic model for the study of profound changes in hybrid plants resulting of expression of various genes. Research of the intensity and orientation of these changes and regularities of ontogenetic development of wheat is necessary to evaluate the viability of hybrids. We have investigated the influence of complementary genes of hybrid dwarfism on the root system, intensity of photosynthesis and the activity of catalase. It was shown that the interaction of complementary dominant genes of hybrid dwarfism has multilateral impact on the ontogenetic development of wheat hybrid Dwarf II. Depression in above ground and underground plant organs was manifested. Photosynthesis in Dwarf II hybrids proceeded more intensively than in the parental forms, but the catalase activity was interrupted in leaves and roots. Notable decrease of the volume and total absorbency of the root surface was detected.
\end{abstract}

Keywords: Wheat, Hybrid Depression, Morphological Parameters, Photosynthesis Intensity, Catalase Activity

\section{Introduction}

The evolution of wheat in some regions of the world have undergone negative dominant mutations, leading to the appearance of depressive or lethal hybrid plants, thereby preventing the success of breeding programs. The divergence of some members of the complementary systems of depression has resulted in their biotypical and ecogeographical localization. Genetic factors in specific combinations have leaded to the interruption of vital activity of regulatory mechanisms, widespread in the genus Triticum. They have diverse manifestations (hybrid necrosis, chlorosis, and hybrid dwarfism), complementary nature and differentiation, depending on species and biotypical and eco-geographical localization $[1,2,3]$. The research of these factors is of great importance, because the knowledge of the changes in physiological traits, associated with genetic gains in yield potential, presents a basic key for understanding the yield-limiting factors and future breeding strategies $[4,5,6]$.

One of the effective approaches in the research of genetic changes in physiological traits is the investigation of three different independent complementary genetic systems (hybrid necrosis, red chlorosis and white spotted chlorosis) leading to the identical physiological changes: disruption of assimilation apparatus, decay of pigments and lower level of viability. Each form of depression has special morphological character, specific development and definitive manifestation. For hybrid dwarfness the intense synthesis and accumulation of chlorophyll is typical. Selection dwarfs wheat cultivars have greater photosynthetic capacities than the taller semi-dwarfs, they averaged $20 \%$ higher maximum net photosynthetic rates compared to the taller semi-dwarfs. The higher rates of photosynthesis are occurring only at anthesis with slightly greater carboxylation efficiencies and significantly increased chlorophyll concentration per unit leaf area [7]. Ellis and Leech [8] stated that the chloroplast population of developing leaf tissues was increased. Depression in hybrids was manifested also on the level of catalase activity in the leaves and roots. The role of dwarf genotypes was specially discussed in the roots formation process, variation in root depth and water extraction [9, 10]. According to Wasson et al. [11] the direct selection of yield leads to the development of varieties with optimal root system. Manschadi et al. [9] stated the importance of root system characteristics to soil exploration and below-ground resource acquisition that are strongly related to plant 
adaptation to sub-optimal conditions.

According to the hypothesis of Hermsen [12], the phenomen of "hybrid dwarfness" is assumed to be determined by the complementary interaction of three genes $D_{1}, D_{2}$ and $D_{3}$, differing in dominance and in contribution to the dwarf phenotype. Depending on the genotype of the parental forms in various stages of development, the hybrids express the features of dwarfness with different levels of viability. Three dwarf types are described [12]. Type 1-dwarfs are dwarf during their whole life cycle and generally do not produce seeds. Type 2-dwarfs are typically dwarf. They have dark green leaves, shortened and thickened stems with productive semisterile ears. In the process of development the hybrid plants are lagging from one of the late maturing parent for 15-20 days. Type 3$d w a r f s$ in the phase of full tillering are forming abundant shoots and light green leaves. At the beginning of phase of earing the hybrid plants are actively growing and almost reaching the level of parental forms. At the end of the growing season the type 3-dwarfs are characterized by abundance of thin stems, small ears and grains.

The objective of our research was the dwarf hybrids physiological traits investigation, as one of approaches for the analysis of gene-to-phenotype relationship.

\section{Materials and Methods}

Our research was conducted on the basis of the Armenian Scientific Center of Agriculture of Ministry of Agriculture, located in Echmiadzin at Ararat region. The region is characterized by dry and sharply continental climate and the cultivation of agricultural crops is conducted under irrigation.

$\mathrm{F}_{1}$ hybrids of wheat were obtained by artificial pollination of the Amby $q$ and Delfi $\hat{\sigma}$ parental forms. Neutered ears were kept in the parchment isolators, then pollinated after 3-5 days. The first hybrid generation $\left(\mathrm{F}_{1}\right)$ seeds and parental lines were sown in $5 \mathrm{~kg}$ pots in triplicate each.

We have determined the changes of some physiological characters of hybrids, compared with parental forms. The intensity of photosynthesis with application of wet combustion technique with minor modifications was used [13]. A disk of tissue from the treated leaf was placed in a small test tube containing $5 \mathrm{ml}$ of chromic acid which was sealed in a $7.5 \mathrm{~cm}$ wide-mouthed McCartney bottle (using a screw cap with a $2,5 \mathrm{~mm}$ thick neoprene seal) containing 2 $\mathrm{ml}$ of $0.2 \mathrm{SN} \mathrm{NaOH}$. The bottles containing leaf disks from the daily samples were autoclaved at $97 \mathrm{kPa}$ for $20 \mathrm{~min}$, and left overnight at room temperature. Complete oxidation of the tissues occured under these conditions, and the released $\mathrm{CO}_{2}$ was absorbed by the $\mathrm{NaOH}$ in the bottles. Loss of water from the $\mathrm{NaOH}$ solution by absorption in the chromic acid reduced the volume of the $\mathrm{NaOH}$ to about $1.7 \mathrm{rnl}$ on average.

The total and working absorbing surfaces of the root system were colorimetrically determined by application of methylene blue according to the method of Sabinin and Kolosov [14].

Estimation of catalase activity, expressed as $\mu \mathrm{M} \mathrm{H}_{2} \mathrm{O}_{2}$ hydrolyzed $\mathrm{mg}^{-1}$ protein $\mathrm{min}^{-1}$ in the plant tissue samples was determined according to the method of Luck [15]. The assay is based on the estimation of residual hydrogen peroxide $\left(\mathrm{H}_{2} \mathrm{O}_{2}\right)$ by oxidation with potassium permanganate $\left(\mathrm{KMnO}_{4}\right)$.

Statistical data processing was performed by Student's ttest.

\section{Results and Discussion}

Completed ontogeny with delayed progress of development for hybrid Dwarf II was detected. The levels of development and morphological parameters of dwarf hybrid completely differed from parental forms.

The morphological differences between hybrid and parental forms are presented in Table 1.

Table 1. The morphological parameters of hybrid Dwarf II and parental forms.

\begin{tabular}{lllll}
\hline Samples & Height of plants, cm & Productive tillering psc/plant & Length of ear, cm & Grain number in ear, psc \\
\hline Amby +9 & $104,90 \pm 1,55$ & $4,20 \pm 0,25$ & $9,10 \pm 0,32$ & $37,0 \pm 1,20$ \\
F $_{1}$ - Dwarf II (Amby x Delfi) & $28,70 \pm 1,07^{*}$ & $5,60 \pm 0,43^{*}$ & $8,10 \pm 0,29^{*}$ & $5,40 \pm 1,80^{*}$ \\
Delfi $\delta^{*}$ & $124,50 \pm 1,62$ & $4,40 \pm 0,16$ & $10,90 \pm 0,24$ & $40,10 \pm 0,93$ \\
\hline
\end{tabular}

Average value \pm standard deviation $(n=3), *$ the differences are significant as compared to the parental forms $(\mathrm{p}<0.05)$

Differences between hybrid Dwarf II and parental forms for all morphological parameters were revealed. Regarding to the height of plants, length of ear and grain number in ear the significantly higher parameters for parental forms were detected. In contrast, the productive tillering of hybrid
Dwarf II was significantly higher in comparison with the parental forms.

Comparative study of capacity of the wheat root system and physiological activity of hybrid Dwarf II and parental forms are presented in Table 2. 
Table 2. Volume and absorbing surface of roots of hybrid Dwarf II and parental forms in the phase of earing.

\begin{tabular}{|c|c|c|c|c|c|c|}
\hline \multirow{2}{*}{ Samples } & \multirow{2}{*}{ Volume of roots, $\mathrm{cm}^{3}$} & \multicolumn{2}{|c|}{ Absorbing surface, $\mathbf{d m}^{2}$} & \multirow{2}{*}{$\begin{array}{l}\% \text { of the working } \\
\text { absorptive surface on total }\end{array}$} & \multicolumn{2}{|c|}{ Surface area } \\
\hline & & Total & Working & & Total & Working \\
\hline Amby + & $7,90 \pm 0,53$ & $1,07 \pm 0,04$ & $0,40 \pm 0,02$ & 38,0 & 1,36 & 0,51 \\
\hline $\mathrm{F}_{1}$ - Dwarf II (Amby x Delfi) & $5,13 \pm 0,20^{*}$ & $0,95 \pm 0,01^{* *}$ & $0,43 \pm 0,01^{* *}$ & 45,40 & 1,85 & 0,84 \\
\hline Delfi $\widehat{O}$ & $8,60 \pm 0,36$ & $1,08 \pm 0,04$ & $0,41 \pm 0,01$ & 38,66 & 1,26 & 0,48 \\
\hline
\end{tabular}

Average value \pm standard deviation $(\mathrm{n}=3), *$ the differences are significant as compared to the parental forms $(\mathrm{p}<0.05),{ }^{* *}$ the differences are insignificant as compared to the parental forms $(\mathrm{p}<0.05)$.

On the base of obtained results the volume of roots of hybrid Dwarf II was significantly lower than in parental forms. Significant differences in absorbing surface were not determined between hybrid Dwarf II and parental forms. The depression, manifested by these parameters was compensated by the increase in percent of the working absorptive surface of roots (45.4\%). The higher level of surface area (total and working) for hybrid Dwarf II was revealed.

The study of the intensity of photosynthesis revealed that even active chlorophyll synthesis in hybrids Dwarf II does not provide their normal viability (Table 3 ).

Table 3. The intensity of photosynthesis in hybrid Dwarf II and its parent forms in earing phase.

\begin{tabular}{lll}
\hline Samples & Intensity of photosynthesis, $\mathbf{~} \mathbf{g} / \mathbf{d m}^{2} / \mathbf{h}$ & The total chlorophyll content, $\mathbf{~ m g / g ~ o f ~ f r e s h ~ w e i g h t ~}$ \\
\hline Amby $q$ & $30,40 \pm 2,23$ & 2,61 \\
F $_{1}$ - Dwarf II (Amby x Delfi) & $42,10 \pm 2,70^{*}$ & $6,21^{*}$ \\
Delfi $\hat{O}$ & $33,10 \pm 1,25$ & 2,70 \\
\hline
\end{tabular}

Average value \pm standard deviation $(\mathrm{n}=3), *$ the differences are significant as compared to the parental forms $(\mathrm{p}<0.05)$.

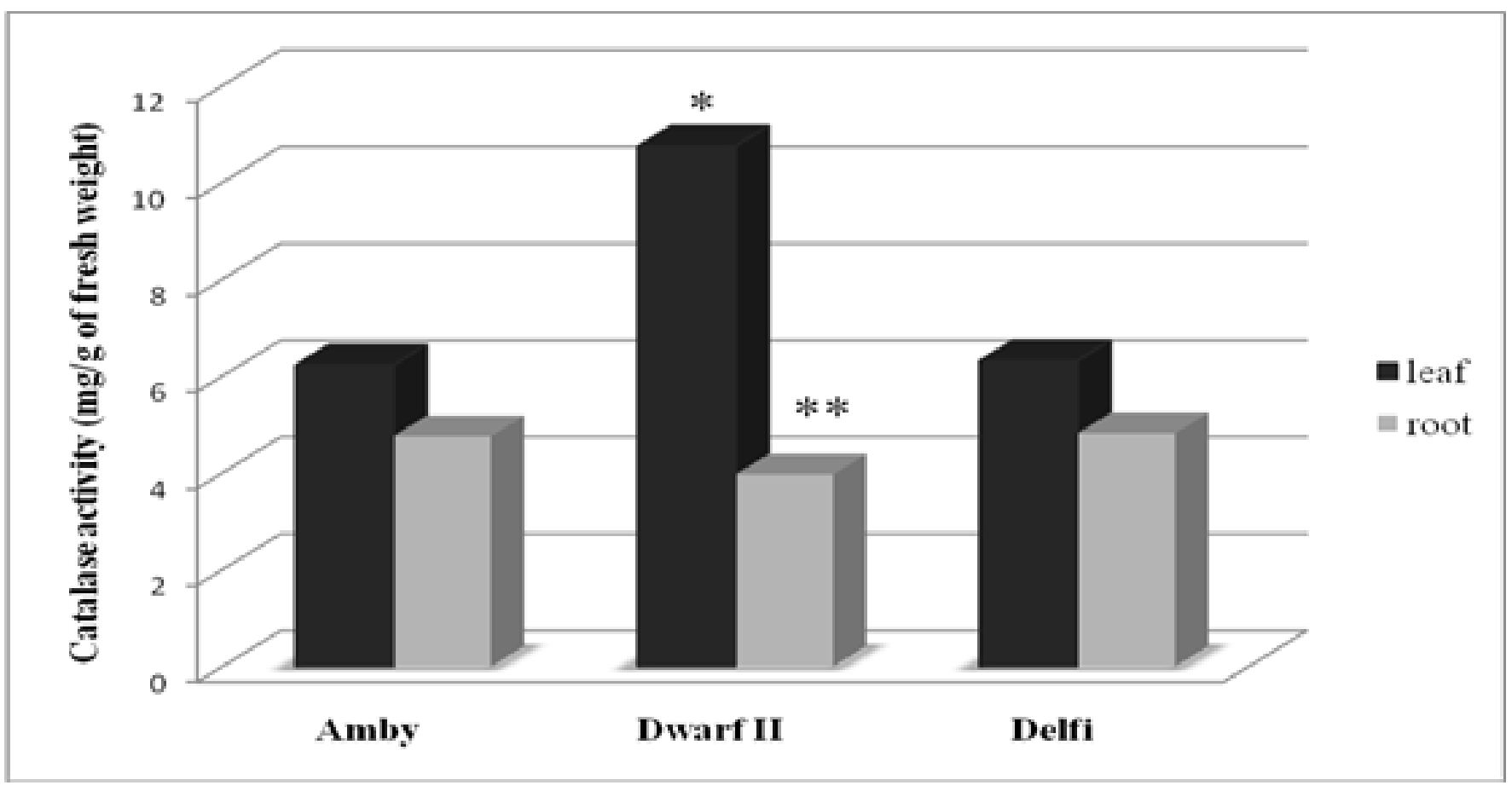

Fig. 1. Catalase activity of leaves and roots in hybrid Dwarf II and in parental forms.

* the differences are significant as compared to the parental forms $(\mathrm{p}<0.05)$,

$* *$ the differences are insignificant as compared to the parental forms $(\mathrm{p}<0.05)$. 
The obtained results were shown, that in the phases of earing the content of total chlorophyll in hybrid Dwarf II was significantly increased $(6.21 \mathrm{mg} / \mathrm{g})$, compared with the parental forms $(2.61$ and $2.70 \mathrm{mg} / \mathrm{g})$.

Our experiments reveal, that the hybrid Dwarf II has a short distance located polar assimilating system- roots and leaves and low level of growth inhibitors. The observed high level of chlorophyll does not provide plants vital activity. Probably, the genetic apparatus of chloroplasts does not ensure realization of the physiological capacity of photosynthetic pigments resulting low levels of chlorophyll activity.

The growth depression was manifested in both aboveground and underground organs. The catalase activity in leaves and roots was also disrupted. The metabolism of root system and, in particular, catalase activity has essential importance for hybrid viability.

As demonstrated in Figure 1 the catalase activity in leaves of Dwarf II was significantly higher, than in the parental forms. The observed differences in the roots between the hybrids and parental forms were insignificant. According to the obtained results the compensatory physiological mechanisms are more effective in the roots than in the leaves. The observed reduction of root capacity and physiological activity of the hybrid can be explained by the increase of working absorptive surface. At the same time, we have shown that closely located assimilating systems, low level of inhibitors of growth processes and high level of chlorophyll of hybrid Dwarf II do not provide normal viability.

Our data are in agreement with the results presented by Bishop and Bugbee [7], when the net photosynthetic rate and chlorophyll concentration were higher in selection dwarf wheat, compared to the taller semi-dwarfs. Earlier Morgan et al. [16] revealed the reduction of leaf cell sizes associated with introduction of semi dwarf stature concentrating the leaf photosynthetic machinery. In turn, the photosynthetic capacity per unit leaf area or leaf weight was increased. Thus, in hybrid Dwarf II photosynthesis occurs more intensively than in parental forms.

\section{Conclusion}

Investigation of the influence of complementary genes of hybrid dwarfism on the ontogenetic development of hybrid Dwarf II revealed significant changes in the root system with the depression of the aboveground organs. Changes of morphological and anatomical parameters, intensity of photosynthesis, catalase activity in leaves and roots and the reduction of of the root power were observed. Elevated level of chlorophyll and increased intensity of photosynthesis did not provide the normal development of genotypically determined hybrid organisms.

\section{References}

[1] Hermsen J. G. (1966). Hybrid necrosis and red hybrid chlorosis in wheat. Hereditas, suppl., 2: 439-452.

[2] Zeven A. C. (1966). Geographical distribution of genes causing hybrid necrosis in wheat. Euphytica, 15(3): 281-284.

[3] Zeven A. C. (1970). Geographical distribution of genes causing hybrid dwarfness in hexaploid wheat of the old world. Euphytica, 19: 33-39.

[4] Edmeadesa G.O., McMasterb G.S., Whitec J.W., Camposa H. (2004). Genomics and the physiologist: bridging the gap between genes and crop response. Field Crops Research 90: $5-18$.

[5] Foulkes M. J., Snape J. W., Shearman V.J., Reynolds M.P., Gaju O., Sylvester-Bradley R. (2007). Genetic progress in yield potential in wheat: recent advances and future prospects, Journal of Agricultural Science 145: 17-29.

[6] Fischer R. A. (2011). Wheat physiology: a review of recent developments Crop and Pasture Science, 62(2): 95-114.

[7] Bishop D.L., Bugbee B.G. (1998). Photosynthetic capacity and dry mass partitioning in dwarf and semi-dwarf wheat (Triticum aestivum L.). J. Plant Physiol. Vt11. 153: 558-565.

[8] Ellis J.R. and Leech R.M. (1985). Cell size and chloroplast size in relation to chloroplast replication in light-grown wheat leaves. Planta 165:120-125.

[9] Manschadi A.M, Christopher J, deVoil P, Hammer GL (2006). The role of root architectural traits in adaptation of wheat to water-limited environments. Functional Plant Biology 33: 823-837.

[10] Palta and Watt. (2009). Vigorous crop root systems: form and function for improving the capture of water and nutrients. In. Crop physiology: applications for genetic improvement and agronomy. Eds V Sadras, D Calderini pp. 309-325.

[11] Wasson A.P., Richards R.A., Chatrath R., Misra S.C., Sai Prasad S.V., Rebetzke G.J., Kirkegaard J.A., Christopher J., Watt M. (2012). Traits and selection strategies to improve root systems and water uptake in water-limited wheat crops. Journal of Experimental Botany, 1-14.

[12] Hermsen J. G. (1967). Hybrid dwarfness in wheat. Euphytica, 16,1: 134-162.

[13] Shimshi D. (1969). A rapid field method for measuring photosynthesis with labeled carbone dioxide. J. Exp. Bot., 20: 381-401.

[14] Tretyakov N.N. (1990). Determination of total and working adsorbing surface of the root system by method of Sabinin and Kolosov. Proceedings on Plant Physiology. Moscow."Agropromizdat", 163-165, (In Russian).

[15] Luck H. (1965). Catalase. In: Bergmeyer, H.U. (Ed.), Method sin Enzymatic Analysis. Academic Press, NY, 885894. 
[16] Morgan J. A., Lecain D. R., Wells R. (1990). Semi dwarfing genes concentrate photosynthetic machinery and affect leaf gas ex-change of wheat. Crop Sci. 30: 602-608. 